\title{
The insulinotropic effect of pulsatile compared with continuous intravenous delivery of GLP-1
}

\author{
Mark P. Plummer ${ }^{1,2} \cdot$ Palash Kar $^{1,2}$ • Caroline E. Cousins ${ }^{2} \cdot$ Kylie Lange $^{3}$. \\ Marianne J. Chapman ${ }^{1,2}$ - Michael A. Nauck ${ }^{4}$ - Michael Horowitz ${ }^{3}$. Juris J. Meier ${ }^{4}$. \\ Adam M. Deane ${ }^{1,2}$
}

Received: 19 October 2015 / Accepted: 6 January 2016/Published online: 30 January 2016

(C) Springer-Verlag Berlin Heidelberg 2016

\begin{abstract}
Aims/hypothesis In healthy individuals, both insulin and glucagon-like peptide 1 (GLP-1) are secreted in a pulsatile fashion. Insulin has greater glucose-lowering properties when administered in pulses compared with a constant i.v. infusion. The primary aim of this randomised double-dummy crossover study was to compare the insulinotropic response to pulsatile and continuous i.v. infusions of equivalent doses of GLP-1.

Methods Twelve healthy participants aged 18-35 years were randomised to three different treatments on separate days: a continuous infusion day (GLP-1 at $0.6 \mathrm{pmol} \mathrm{kg}^{-1} \mathrm{~min}^{-1}$ [1 $\mathrm{ml} / \mathrm{min}$ ] and a $1 \mathrm{ml}$ placebo bolus every $6 \mathrm{~min}$ ); a pulsatile infusion day (placebo at $1 \mathrm{ml} / \mathrm{min}$ and a $3.6 \mathrm{pmol} / \mathrm{kg}$ GLP-1 bolus every $6 \mathrm{~min}$ ); and a placebo day (placebo at $1 \mathrm{ml} / \mathrm{min}$ and a $1 \mathrm{ml}$ placebo bolus every $6 \mathrm{~min}$ ). Between 45 and $120 \mathrm{~min}$, a hyperglycaemic clamp was used to maintain blood glucose at $9 \mathrm{mmol} / \mathrm{l}$. Venous blood glucose and plasma insulin concentrations were measured every $5 \mathrm{~min}$ from 0 to $45 \mathrm{~min}$ and every $1 \mathrm{~min}$ from 45 to $120 \mathrm{~min}$; plasma glucagon was measured every $15 \mathrm{~min}$. The order of treatment was randomised by the
\end{abstract}

Mark P. Plummer

mark.plummer@adelaide.edu.au

1 Discipline of Acute Care Medicine, University of Adelaide, Adelaide, SA, Australia

2 Intensive Care Research Unit, Level 4 Emergency Block, Royal Adelaide Hospital, North Terrace, Adelaide, SA 5000, Australia

3 Discipline of Medicine, University of Adelaide, Adelaide, SA, Australia

4 Division of Diabetes and GI Endocrinology, University Hospital St Josef-Hospital, Ruhr-University Bochum, Bochum, Germany
Pharmacy Department and both study investigators and participants were blinded to the treatment arm. The dextrose requirement and glucagon data were analysed using repeated measures ANOVA and insulin data were analysed with a linear mixed effects maximum likelihood model.

Results Continuous and pulsatile infusions of GLP-1 increased the dextrose requirement by $\sim$ threefold $(p<0.001)$ and increased insulin secretion by $\sim$ ninefold $(p<0.001)$. There was no difference in the effect of both treatments. Although hyperglycaemia reduced plasma glucagon concentrations, there was no difference between the treatment days. Conclusions/interpretation In healthy individuals, pulsatile and continuous administration of i.v. GLP-1 appears to have comparable insulinotropic effects.

Trial registration: ACTRN12612001040853

Funding: This study was supported by the National Health and Medical Research Council (NHMRC) of Australia.

Keywords Glucagon · Glucagon-like peptide 1 - Insulin · Pulsatile

\section{Abbreviation \\ GLP-1 Glucagon-like peptide 1}

\section{Introduction}

In healthy individuals, a pulsatile pattern of hormone secretion is a fundamental property of a number of endocrine functions, including those of the parathyroid, pituitary, adrenal and islet cell hormones [1]. It is well established that, in addition to basal release, the majority of insulin is secreted in discrete high-frequency bursts [1]. This oscillatory pattern of insulin secretion is pivotal to optimal insulin action. In type 2 
diabetes, attenuation of the secretory burst mass and a loss of pulsatile orderliness are prominent pathophysiological features [2]. In healthy and in type 1 diabetic individuals, i.v. insulin has a more pronounced glucose-lowering effect when given in a pulsatile, rather than a continuous, fashion [3].

The incretin hormone glucagon-like peptide 1 (GLP-1) lowers blood glucose via the glucose-dependent stimulation of insulin and inhibition of glucagon secretion, and via slowing gastric emptying [4]. Balks and colleagues reported that GLP-1 is secreted in a pulsatile manner during both basal and glucose-stimulated conditions, with a pulse interval of $\sim 7 \mathrm{~min}$ [5]. However, the effect of exogenous GLP-1 and GLP-1 agonists has hitherto only been determined using continuous i.v. or subcutaneous infusions, and the effect of pulsatile i.v. administration has not been assessed.

The primary aim of this study was to determine whether i.v. pulsatile administration of GLP-1 increased insulin secretion during euglycaemia and hyperglycaemia when compared with continuous administration.

\section{Methods}

Participants Healthy participants aged 18-35 years were considered eligible and attended a screening visit at the Royal Adelaide Hospital. Written informed consent was obtained from all participants. The study protocol was approved by the hospital's Research Ethics Committee and prospectively registered (www.anzctr.com.au; ACTRN12612001040853).

Protocol All participants underwent three different treatments on different days separated by a minimum of 7 days. Treatment days comprised: a control or continuous infusion day (continuous infusion of GLP-1 at $0.6 \mathrm{pmol} \mathrm{kg}^{-1} \mathrm{~min}^{-1}$ [1 $\mathrm{ml} / \mathrm{min}$ ] and a $1 \mathrm{ml}$ bolus of $4 \%$ human albumin every $6 \mathrm{~min}$ ), an intervention 'pulsatile' day (continuous infusion of $4 \%$ albumin and a $1 \mathrm{ml}$ bolus of $3.6 \mathrm{pmol} / \mathrm{kg}$ GLP-1 every $6 \mathrm{~min}$ ) and a placebo day (continuous infusion of $4 \%$ human albumin and a $1 \mathrm{ml}$ bolus of $4 \%$ albumin every $6 \mathrm{~min}$ ). Each treatment period lasted $2 \mathrm{~h}$ and a total dose of $72 \mathrm{pmol} / \mathrm{kg}$ GLP-1 was delivered on both control and intervention days. On each treatment day, a hyperglycaemic clamp [6] was commenced at $t=45 \mathrm{~min}$ to obtain a target blood glucose level of $9 \mathrm{mmol} / \mathrm{l}$ that was maintained until study completion at $t=120 \mathrm{~min}$. The order of treatment was randomised by the Pharmacy Department and both study investigators and participants were blinded to the treatment arm.

Participants attended the Royal Adelaide Hospital at 08:30 after an overnight fast. Two i.v. cannulae were inserted into the left arm of each participant: one into the cubital fossa for an infusion of $25 \%$ dextrose and the other into the hand for infusion of the study drug. A third i.v. cannula was inserted into the right cubital fossa for blood sampling. Synthetic GLP-1 amide
(Bachem, Weil am Rhein, Germany) was reconstituted by the Pharmacy Department in 4\% albumin and presented in i.v. bags (containing $120 \mathrm{ml}$ ) for infusion at $0.6 \mathrm{pmol} \mathrm{kg}^{-1} \mathrm{~min}^{-1}$ or in sets of $20 \times 1 \mathrm{ml}$ syringes each containing a dose of $3.6 \mathrm{pmol} / \mathrm{kg}$ such that placebo and GLP-1 treatments looked identical. After drawing baseline blood samples, the first bolus was given, followed by a $2 \mathrm{ml}$ flush of $0.9 \%$ saline. Continuous infusion at $1 \mathrm{ml} / \mathrm{min}$ was started concurrently and maintained for the duration of the study. Bolus administration was repeated as described every 6 min until study completion at $t=120 \mathrm{~min}$. Commencing at $t=0 \mathrm{~min}$, venous blood samples for measuring glucose, insulin and glucagon were taken at $5 \mathrm{~min}$ intervals until $t=45 \mathrm{~min}$, and then every minute until $t=120 \mathrm{~min}$. The total amount of $25 \%$ dextrose required to maintain target blood glucose levels on each day was recorded.

Laboratory measurements Glucose was measured using a portable electrochemical coulometric glucose dehydrogenase glucose meter with a CV of 3.8\% in the target range (Optium Xceed; Abbott Laboratories, Abbott Park, IL, USA).

Once clotted, blood samples were centrifuged at 3,147 $\mathrm{g}$ for $15 \mathrm{~min}$ and serum was stored at $-70^{\circ} \mathrm{C}$ for the subsequent measurement of insulin and glucagon. Insulin was analysed using an ultrasensitive paramagnetic immunoassay (Access Immunoassay Systems, Beckman Coulter, Brea, CA, USA) that has an analytical sensitivity of $0.21 \mathrm{pmol} / 1$ and an interassay CV of $2.98 \%$. Immunoreactive glucagon was measured by RIA (GL-32K; Millipore, Germany) that has an analytical sensitivity of $20 \mathrm{pg} / \mathrm{ml}$, an intra-assay CV of $3.8 \%$ and an interassay $\mathrm{CV}$ of $8.2 \%$.

Statistical analysis The overall effects of insulin and glucagon were calculated as the AUC. Demographic and glucose data are means (SD) and insulin and glucagon data are means (SEM). Dextrose requirements and glucagon AUCs were evaluated using repeated measures ANOVA with Bonferroni correction; insulin AUCs were analysed using a linear mixed effects maximum likelihood model with a fixed effect for treatment, an unstructured covariance matrix to account for repeated visits per participant and Bonferroni-adjusted tests for pairwise post hoc comparisons. Statistical analyses were performed using IBM SPSS (version 22.0; Chicago, IL, USA).

\section{Results}

Twelve healthy participants (nine men, aged 22 [2.6] years, BMI $24[2.4] \mathrm{kg} / \mathrm{m}^{2}$ ) completed all three treatment days without significant adverse events.

Blood glucose levels During the euglycaemic period $(t=0$ $45 \mathrm{~min}$ ), blood glucose decreased by $\sim 1 \mathrm{mmol} / 1$ on both the continuous and pulsatile GLP-1 infusion days and remained 
stable on the placebo day. Blood glucose concentrations were effectively clamped at the hyperglycaemic target on the placebo $(9.0[0.2] \mathrm{mmol} / \mathrm{l})$, continuous GLP-1 $(8.9[0.5] \mathrm{mmol} / \mathrm{l})$ and pulsatile GLP-1 $(8.8[0.2] \mathrm{mmol} / \mathrm{l})$ infusion days. Dextrose requirements to maintain the hyperglycaemic clamp were approximately threefold greater on both the continuous and pulsatile GLP-1 infusion days compared with the placebo days $(p<0.001)$. There was no significant difference in dextrose requirement between the continuous and pulsatile GLP-1 infusion days $(p=1.0)$.

Serum insulin levels In all participants, insulin concentrations approximated a steady plateau from $0 \mathrm{~min}$ to $45 \mathrm{~min}$. There was an abrupt rise after commencement of the hyperglycaemic clamp, with a return to a higher plateau for the placebo and a progressive linear increase for both pulsatile and continuous GLP-1 infusion (Fig. 1). Insulin concentrations were greater both during the euglycaemic period $(t=0-45 \mathrm{~min})$ and overall with both continuous and pulsatile infusions of GLP-1 than with placebo $(p<0.001$; Fig. 1). There was no difference in insulin concentration between the continuous and pulsatile GLP-1 infusion days $(p=1.0)$.

Serum glucagon levels For all treatments, glucagon concentration remained stable until commencement of the hyperglycaemic clamp, following which there was a progressive decline from $45 \mathrm{~min}$ to $120 \mathrm{~min}$. There was no overall difference in glucagon suppression among all three treatment days (Fig. 2).

\section{Discussion}

The key observation of this study is that during both euglycaemia and hyperglycaemia there was no difference in

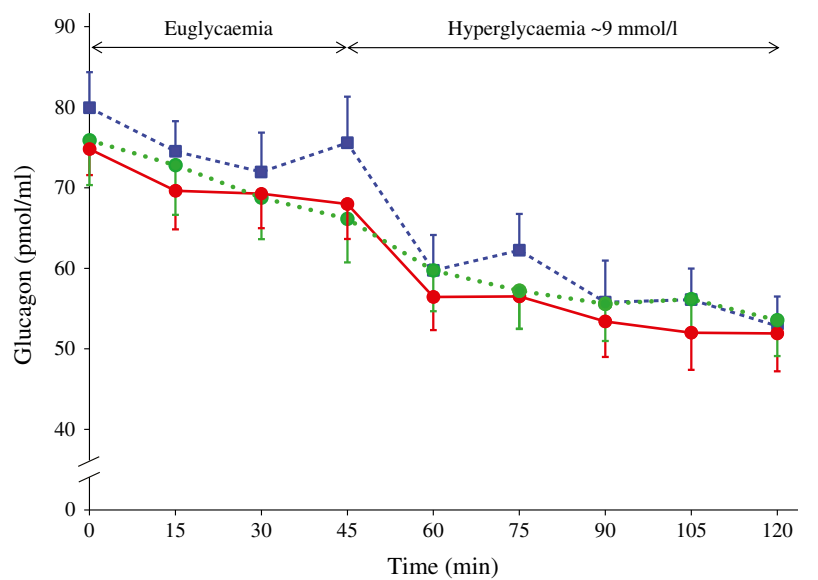

Fig. 2 Effect of GLP-1 infusion on serum glucagon concentrations. There was no difference in plasma glucagon across all treatment days during both euglycaemia $(t=0-45 \mathrm{~min})$ and hyperglycaemia $(t=45$ $120 \mathrm{~min}$ ). Red circles, continuous GLP-1 infusion; blue squares, pulsatile GLP-1 infusion; green circles, placebo. Data are means \pm SEM

the insulinotropic response to pulsatile i.v. delivery of GLP-1 compared with continuous administration of an equivalent dose $\left(0.6 \mathrm{pmol} \mathrm{kg}^{-1} \mathrm{~min}^{-1}\right)$.

Our study is the first to investigate the insulinotropic efficacy of replicating physiological pulsatile GLP-1 secretion with the administration of exogenous peptide at supraphysiological concentrations. Prior studies utilising i.v. or subcutaneous infusions of GLP-1 in healthy and diabetic individuals have exclusively delivered GLP-1 as a continuous infusion and, of more clinical relevance, commercially available GLP-1 agonists (with a subcutaneous route of delivery) produce similarly stable plasma concentrations [7]. Data from the present study suggest that the pulsatile delivery of GLP-1 is unlikely to result in a greater insulinotropic response.

Both i.v. glucose infusions and exogenous GLP-1 suppress endogenous glucagon secretion, and the effect of GLP-1 is

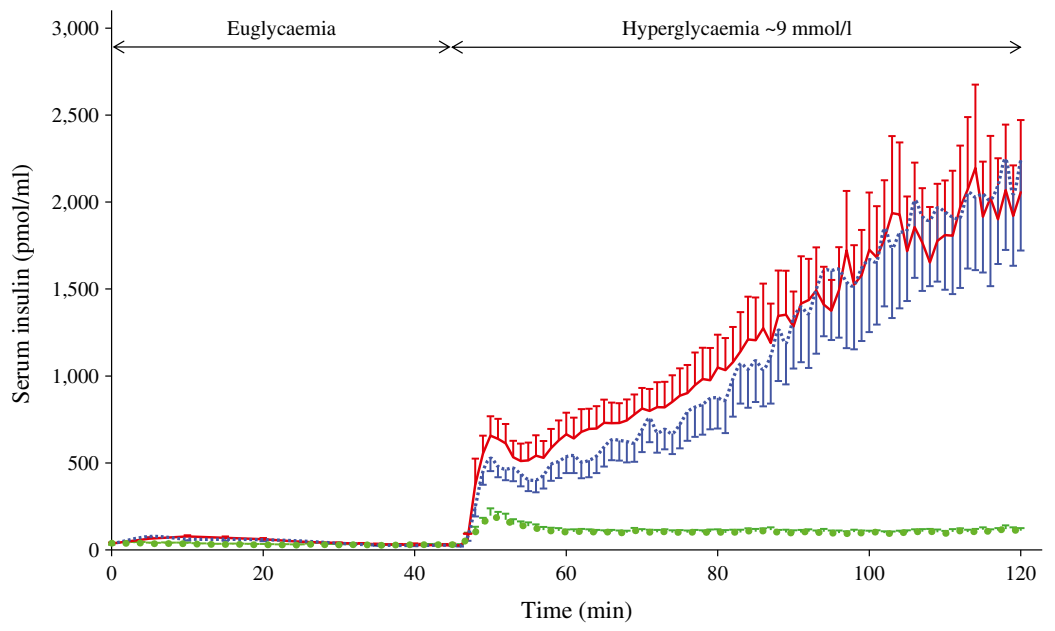

Fig. 1 Effect of GLP-1 infusion on serum insulin concentrations. Continuous (red solid line) and pulsatile (blue dashed line) infusions of GLP-1 increased serum insulin by $\sim 50 \%$ compared with placebo (green dashed line) during euglycaemia $(t=0-45 \mathrm{~min} ; p<0.001)$. Overall serum insulin was increased $\sim$ ninefold during continuous and pulsatile infusions of GLP-1 compared with placebo $(p<0.001)$. There was no difference in serum insulin levels between continuous and pulsatile GLP-1 infusion regimens $(p=1.0)$. Data are means \pm SEM 
glucose dependent [4]. In the present study, there was no difference in the magnitude of glucagon suppression during hyperglycaemia regardless of placebo or GLP-1 administration, suggesting that in healthy individuals glucagon suppression is maximal following an i.v. hyperglycaemic clamp.

The limitations of this study should be recognised. Based on physiological secretion, only a single interval $(6 \mathrm{~min})$ of pulses was tested, and a different duration may potentially change the effect. A continuous GLP-1 dose of $3.6 \mathrm{pmol} / \mathrm{kg}$ (at $0.6 \mathrm{pmol} \mathrm{kg}^{-1} \mathrm{~min}^{-1}$ ) was selected because the equivalent bolus dose is above the threshold known to have an insulinotropic effect and below the dose likely to have adverse effects [8]. However, we cannot exclude the possibility that lower or higher doses may have an adverse effect or whether a 'ceiling' effect to i.v. GLP-1 boluses occurs, as seen for other G protein-coupled linked pathways [9]. Hyperglycaemia markedly potentiates the insulinotropic response to GLP-1 in healthy participants [10]. For this reason, a 'moderate' glycaemic clamp of $9 \mathrm{mmol} / \mathrm{l}$ was utilised to minimise the synergistic effects of exogenous glucose, but we did not test multiple glucose concentrations. Further, we studied healthy volunteers and cannot exclude that the response may be different in the setting of diabetes. Finally, we did not measure GLP-1 levels and, accordingly, are unable to comment on whether pulses administered peripherally lead to a difference in pulse amplitude or overall GLP-1 concentration in either plasma or the portal bed compared with continuous delivery.

In conclusion, this study indicates that pulsatile and continuous i.v. infusions of GLP-1 have comparable insulinotropic properties and thus that pulsatile delivery regimens of GLP-1 or its agonists are unlikely to have greater glucose-lowering effects. However, the effects of different pulse intervals and doses of GLP-1 warrant investigation.

Funding This study was supported by Project Grant 1025648 from the National Health and Medical Research Council (NHMRC) of Australia. MPP is supported by an NHMRC postgraduate scholarship and AMD is supported by an NHMRC Early Career Fellowship.

Duality of interest statement JJM has served on advisory boards for, received honoraria or consulting fees from, or received research funding from AstraZeneca, Berlin-Chemie, Bristol-Myers Squibb, Boehringer Ingelheim, Eli Lilly, MSD, Novo Nordisk, Novartis, Roche and SanofiAventis. MAN has served on advisory boards for, received honoraria or consulting fees from, or received research funding from Amylin, AstraZeneca, Berlin-Chemie, Boehringer Ingelheim, Bristol-Myers Squibb, Diartis Pharmaceuticals, Eli Lilly, GlaxoSmithKline, Hoffmann-La Roche, Intarcia Therapeutics, Janssen, MannKind,
Merck, MetaCure, Novartis, Novo Nordisk, Roche Pharmaceuticals, Sanofi, Takeda, Versartis and Wyeth Research. MH has participated in advisory boards and/or symposia for AstraZeneca, Boehringer Ingelheim, Eli-Lily, Meyer Nutriceuticals, Novartis, Novo Nordisk, Sanofi-Aventis and Satiogen Pharmaceuticals. All other authors declare that there is no duality of interest associated with their contribution to this manuscript.

Contribution statement MPP, JJM and AMD were responsible for study conception and design and PK, MAN, MJC and MH contributed to the study design; AMD was responsible for obtaining funding; MPP, $\mathrm{AMD}$ and $\mathrm{CEC}$ were responsible for data acquisition; MPP and KL were responsible for statistical analysis; MPP, MAN, MH, JJM and AMD were responsible for data interpretation; MPP drafted the manuscript; all other authors critically revised the manuscript for important intellectual content; and all authors approved the final version to be published. MPP is the guarantor of this work and, as such, had full access to all the data in the study and takes responsibility for the integrity of the data and the accuracy of the data analysis.

\section{References}

1. Porksen N (2002) The in vivo regulation of pulsatile insulin secretion. Diabetologia 45:3-20

2. Veldhuis JD, Keenan DM, Pincus SM (2008) Motivations and methods for analysing pulsatile hormone secretion. Endocr Rev 29:823-864

3. Paolisso G, Sgambato S, Torella R et al (1988) Pulsatile insulin delivery is more efficient than continuous infusion in modulating islet cell function in normal participants and patients with type 1 diabetes. J Clin Endocrinol Metab 66:1220-1226

4. Deacon CF, Ahren B (2011) Physiology of incretins in health and disease. Rev Diabet Stud 8:293-306

5. Balks HJ, Holst JJ, von zur Muhlen A, Brabant G (1997) Rapid oscillations in plasma glucagon-like peptide1 (GLP-1) in humans: cholinergic control of GLP-1 secretion via muscarinic receptors. J Clin Endocrinol Metab 82:786-790

6. Plummer MP, Jones KL, Cousins CE et al (2015) Hyperglycaemia potentiates the slowing of gastric emptying induced by exogenous GLP-1. Diabetes Care 38:1123-1129

7. Lund A, Knop FK, Vilsboll T (2014) Glucagon-like peptide1 receptor agonists for the treatment of type 2 diabetes: differences and similarities. Eur J Intern Med 25:407-414

8. Kjems LL, Holst JJ, Volund A, Madsbad S (2003) The influence of GLP-1 on glucose-stimulated insulin secretion: effects on beta cell sensitivity in type 2 and nondiabetic participants. Diabetes 52:380 386

9. Christopoulos A, Kenakin T (2002) G protein-coupled receptor allosterism and complexing. Pharmacol Rev 54:323-374

10. Vilsboll T, Toft-Nielsen MB, Krarup T, Madsbad S, Dinesen B, Holst JJ (2000) Evaluation of beta cell secretory capacity using glucagon-like peptide 1. Diabetes Care 23:807-812 